\title{
Über die verschiedenen Fassungen der Bertasage.
}

\author{
(S. Zeitschr. XXXV, I.)
}

Bei der Besprechung des Verhältnisses der durch die Reali überlieferten Version zu anderen Werken, die die Bertasage enthalten, gelangt P. Rajna zu dem Schlusse, dals ihr Verfasser $V, A$ und überdies noch eine dritte, heute verschollene Fassung benutzte. G. Paris lehnt $a$ priori Adenet als Quelle des Andreas da Barberino ab, denn seiner Ansicht nach üben die französischen Epen aus der zweiten Hälfte des XIII. Jahrh., d. i. der Verfallszeit, bereits keinen Einflufs mehr auf die italienische Literatur aus. Feist verwirft, seinem Grundsatze getreu, nicht nur den brabantischen Dichter, sondern auch den franko-italienischen und behält jene nicht näher bekannte Version, die offenbar verschieden ist von der verschollenen, die dem franko-italienischen Dichter als Muster diente, und verschieden auch von der, die Adenet benutzte.

Hat Feist's Hypothese keinerlei Argumente für sich, so lassen sich andererseits mehrere Tatsachen anführen, die geradezu ihre Wahrscheinlichkeit ausschliefsen. Es unterliegt keinem Zweifel, dafs kein französischer Dichter die Gesandten von Paris nach Ungarn durch die Lombardei führen würde. Der gewöhnliche Weg führte über Deutschland, wie man es bei Adenet sieht. Wenn wir in dem franko-italienischen Gedichte lesen:

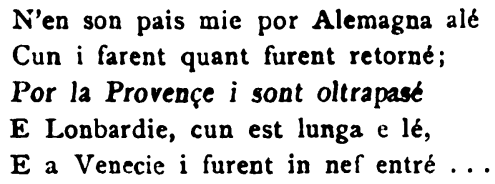

$$
\text { (V. 3II-I5.) }
$$

so spricht daraus deutlich der Lokalpatriotismus. Aber der frankoitalienische Dichter mufste wenigstens einmal die Gesandten über Deutschland führen, nämlich, als sie mit Berta nach Paris reisen, denn die ungarische Königstochter sollte die Tochter des Grafen von Mainz mitnehmen.

Wenn also die Reali die französischen Gesandten desselben Weges ziehen lassen, so scheint ihre Abhängigkeit von dem frankoitalienischen Gedichte keinem Zweifel zu unterliegen. Und tat-

Zeitschr. f. rom. Phil. XXXV. 
sächlich haben die beiden Fassungen nicht nur denselben Verlauf der Handlung, sondern stimmen auch in vielen Einzelheiten überein und man kann sogar wörtliche Reminiszenzen finden, auf die P. Rajna in seiner Arbeit aufmerksam gemacht hat.

Aber der Verfasser von $R$ kannte das Gedicht des Adenet. Man kann dafür zahlreiche Beweise anführen. Die wichtigsten zählte ebenfalls P. Rajna auf, indem er die Ähnlichkeiten beider Werke hervorhob. Es muls aber noch ein von dem brabantischen Dichter übernommenes Motiv in $R$ berücksichtigt werden. Wir wissen, dafs bei Adenet Simons Gattin den unbekannten Ritter wegen seines ungebührlichen Benehmens Berta gegenüber im Walde mit heftigen Worten ausschilt, ferner, dals Berta, zum Unterschiede von allen anderen Versionen, ihre Frauenehre mit voller Würde verteidigt. Der brabantische Dichter allein betonte diese moralische Seite und ging darin so weit, dafs er in diesem Punkte den ursprünglichen Verlauf der Handlung änderte. Barberino sah sich nun vor zwei grundverschiedenen Fassungen. Nach der einen gehen sowohl die Beschützer als auch die Heldin selbst leichten Herzens auf die Gelüste des Königs ein, nach der andern treten sie mit aller Würde auf. Um beide Überlieferungen zu vereinigen, kompiliert der Verfasser von $R$ beide auf eine sehr ungeschickte Weise, indem er Bertas Beschützern die Würde verleiht, die bei Adenet die Heldin auszeichnet. Auf die Kunde, dals sich Berta Pipin willig hingibt, überschüttet sie Lamberto mit einem Hagel von Epitheten, die sich nicht wiederholen lassen; dieses Vorgehen ist jedoch nicht am Platze und zeugt zugleich von der Ungeschicklichkeit des Verfassers bei der Vereinigung zweier einander widersprechender Überlieferungen.

So haben also die Reali, als eine Version von erhaltenen Werken, 1 keinen Wert für die Rekonstruktion des ursprünglichen Gedichtes. Aber auch hinsichtlich des literarischen Wertes erhebt sich die Erzählung des Andreas Barberino nicht über das Mittel-

1 Wenn P. Rajna noch eine verschollene Version als dritte Quelle der Reali di Francia annehmen $2 u$ müssen glaubt, weil ihm manche Züge in $R$ archaistisch vorkommen und vor allem, weil sowohl in $R$ als in $T$ Beria den König sogleich erkennt, so ist dagegen zu bemerken, dals dieses Motiv nur zwei Lösungen hat: entweder Berta erkennt den König, oder sie erkennt ihn nicht. Die Ăhnlichkeit eines solchen Zuges in 2 wei Versionen kann rein zufallig sein und beweist noch lange nicht die Existenz einer gemeinsamen Quelle. Dabei lärst sich in $R$ und $T$ diese Ähnlichkeit aus der ähnlichen Tendenz der Verfasser, die Erzählung zu kürzen, genügend erklären. In $T$ ist schon die Königinmutter längst abgereist. Pipin, der incognito zum Hirten kam, fragt Berta, wer sie sei, und da sie den König gut kannte (et cela qui bien le connoisset), so erzählt sie ihm ibre Schicksale und die Geschichte nimmt damit ein Ende. Anders in $R$. Pipin kommt als König zu Lamberto, es ist daher nichts Auffallendes, wenn Berta weils, wer er ist, - alle wissen es $\mathrm{ja},-$ und da ihre Eltern im Begriff sind abzureisen, so ergreift der Verfasser selbstverständlich die Gelegenheit, die er selbst hervorgerufen hat, um ihnen eine $z$ weite Reise zu ersparen und lälst Berta dem Könige alles erzählen, sonst müfste er, wie bei Adenet, noch einmal Bertas 
mälsige. Vergebens würde man bei ihm eine erhebende Szene oder ein ausdrucksvolleres Bild suchen. $Z u$ den einigermalsen gelungenen Motiven gehört das Flehen der Menge für die Kinder der falschen Königin, zu den satirischen die Furcht Pipins vor dem Schwiegervater, er könnte sein Verhältnis zu Berta merken.

Das dritte Werk in Italien, das die Bertasage enthält, ist ein frankoitalienischer Prosaroman, bisher noch nicht in Druck erschienen, dessen Verfasser ein gewisser Rafael Marmora zu sein scheint. 1

Roland, der unter phantastischen Abenteuern die halbe Welt und viele wunderbare Dinge, unter andern auch das Pferd Salvaze, einen Nachkommen in gerader Linie von Alexanders Bukephalos, erobert hat, verläfst auf Gottes Befehl heimlich die Stadt Montlion und macht sich mit Trepin auf die Suche nach dem Kaiser, der hinterlistig von den Feinden des Kreuzes entführt worden war. Das Ziel ihrer Reise ist das Schlofs Valperse, in dem der eingekerkerte Charlemagne mit vier andern christlichen Königen weilte. Als sie unterwegs an einem hohen Berge vorbeigehen, hören sie Geschrei und Stöhnen, das aus der Tiefe kommt. Roland allein wagt es, ins Innere einzudringen und es zeigt sich, da/s hier das Fegefeuer ist. Er trifft dort die Seele seiner Grolsmutter an, die so hinterlistig an Berta gehandelt hatte. Dafür bülst sie schwer, hofft jedoch, dafs jetzt alles enden und sie, durch Bufse gereinigt, an den ihr bestimmten Ort kommen werde. Wie einst die Schatten der Unterwelt dem Odysseus, so erzählt sie ihrem Enkel verschiedene Begebenheiten, denen Roland andächtig zuhört. Schliefslich fragt er, von Neugier getrieben, wieso sie für Berta gehalten werden konnte. Habe sie denn niemand gekannt und niemand die ungarische Königstochter? Man könne doch unmöglich annehmen, dafs niemand den Betrug durchschaut habe. „Die Geschichte klingt mir märchenhaft", würde Roland sagen, lebte er in unserem Zeitalter. So könne sich die Sache nicht verhalten haben. ${ }_{n}$ Du hast Recht", antwortete da Gaiete - denn diesen Namen beliebte es dem Verfasser der Grolsmutter Rolands und Freundin Bertas zu geben "daran ist kein Wort wahr" (zu ergänzen: was die Jongleurs erzählen). Pipin sah doch seine Gemahlin den ganzen Tag hindurch, am Hofe weilten doch die Gesandten Aquilon de Bavière, Grifon d'Altefogie, Quintin de Normandie, Morand de Rivere. Sie waren doch alle in Ungarn, kannten Berta gut und kannten sehr wohl auch mich. Nein, so verhielt sich die Sache nicht. Wenn du zuhören willst, werde ich dir erzählen, wie es geschah, damit du die Wahrheit erfahren und sie deiner Mutter und anderen mitteilen könnest.

Eltern herbeibringen. Wir haben also keinen Grund eine verlorene oder dritte Version als Quelle der Reali anzunehmen.

1 Siehe Romania XI (S. $536 \mathrm{ff}$.), wo A. Thomas eine treffliche Analyse und einige wichtige Stellen in extenso veröffentlicht hat. 
Nachdem man den Inhalt der neuen Version kennen gelernt hat, drängt sich unwillkürlich der Gedanke auf, ob es nicht die franko-italienische Kompilation war, die der Verfasser als Quelle benutzt hatte.1 Auf den ersten Blick zwar scheinen die beiden Fassungen sehr wenig gemeinsame Züge zu haben. Fehlt ja doch in Marmora's Erzählung von Berta das wesentlichste Merkmal des franko-italienischen Gedichtes, es fehlt das Geschlecht der Mainzer. Die falsche Königin stammt nicht aus dieser Verräterfamilie, sondern ist einfach eine kroatische Königstochter. Aber das ist nur scheinbar. Nimmt man die übrigen Teile des Romans näher in Augenschein, so sieht man dort die Mainzer Verräterfamilie in fortwährenden blutigen Kämpfen mit den treuen Vasallen des Kaisers. Als der heidnische König Lucion Karl den Grofsen fortgeschleppt und Roland sich auf die Suche begeben hatte, rifs Gaine aus dem Mainzer Geschlechte die Regierung in Paris an sich. Damals kam es zum Kampfe mit den Anhängern Clermonts, in dem Grifon und einige andere Mainzer fielen. Nach der Rückkehrt gestattete der Kaiser dem Rainald und a la geste de Clermont, die Maganzois gänzlich auszurotten. Marmora kennt also genau den Verlauf der ganzen Handlung in der franko-italienischen Kompilation und spinnt sie auf seine Art weiter. Weshalb machte er aber die falsche Königin nicht zur Mainzer Gräfin? Wäre es nur eine Kaprice? Das ist leicht $\mathrm{zu}$ bejahen, aber wie soll man es erklären? Mir scheint, es liefse sich ein Grund dafür finden, und zwar ein ziemlich schwerwiegender. Nach den französischen Werken hatte die falsche Königin Pipin zwei Söhne geschenkt, nach der venetianischen Handschrift überdies noch eine Tochter, die Milon heiratete und Roland gebar. Damit würde Roland zu dem Mainz'schen Geschlechte gehören, demnach zu einer Verräterfamilie, er, die treueste Stütze des Throns. $\mathrm{Zu}$ der Zeit, wo der franko-italienische Dichter seine Kompilation schrieb, war eine solche Einreihung Rolands in dieses Geschlecht noch nicht kompromittierend, da doch der Dichter erst selbst jene Verräterfamilie schuf und nicht merkte oder auch nicht zu merken brauchte, welchen Lapsus er beging. Aber die Zeiten änderten sich. Der Name der Mainzer war in der Literatur Norditaliens verunglimpft. Es wäre linerhört, aus diesem Geschlechte Roland, den populärsten Helden Italiens, stammen zu lassen. Andererseits sagte ihm die Quelle ausdrücklich, dals Rolands Grolsmutter die falsche Königin war. Und daher machte er mit einem Federzuge die Gräfin von Mainz zur kroatischen Königstochter.

Gehen wir weiter. Der venetianischen Handschrift zufolge. ist Rolands Mutter, als Tochter der Verräterin, die hinterlistig Bertas Platz einnahm, ein im Konkubinate gezeugtes Kind, da doch die im Namen Pipins geschlossene Trauung sich auf eine andere Person bezog. Und ein solcher Makel durfte doch nicht auf

1 A. Thomas glaubt, dafs ein verlorenes Gedicht seine Quelle war. Romania XI, S. 545 . 
einem Helden lasten, der ein Liebling des italienischen Volkes geworden war, das ihn fast über drei Jahrhunderte besang, und der endlich auch die Hauptperson in Marmora's Romane ist. Er mufste rein gewaschen werden. Es folgte die zweite Änderung. Nicht durch Betrug, sondern offen, vor aller Augen wurde die hinterlistige Freundin Pipins Gattin. Die andere entfloh, starb vielleicht sogar. Der Platz für sie ist frei. Die Tendenz des Verfassers spricht deutlich aus den Worten Gaiete's an Roland. Zweimal betont or es. Einmal sagt es Pipin zu Gaiete: "Gi vos torai a dame, si vos sposerai por tal convenant si por aucun temp la (Berte) trovasse gi la farai ardre e pois ferai confermer le matrimonie da toi a moi da li apostolice de Rome, a ce che se feistes fil ne file che soient legitime a poir rediter la corone de Franze". Sodann versichert Gaiete selbst Roland, dafs seine Mutter ein eheliches Kind war: „e si aui de lui dui fil, ce fu Lanfroi e Lodris, e de pois une file, ce fu Berte toe mere, e voiremant por sapue de pere e de mere ta mere non fue bastarde." Deutlicher kann man nicht sein und die Absicht des Dichters tritt ganz klar hervor.

So erklären sich also die zwei prinzipiellen Unterschiede aus der Tendenz des Romans. Sie haben gänzlich das Aussehen dieser Version verändert und bewirkt, dals diese so wenig dem frankoitalienischen Gedichte ähnlich ist. Es gibt jedoch Spuren dieser Umarbeitung, die keinen $Z$ weifel hinsichtlich der Abstammung der Version Marmora's übrig lassen. Wir wissen bereits, dals der franko-italienische Dichter die alte Dienerin und die betreffende Szene beseitigte und dafür ein ganz albernes und unlogisches Motiv erdachte, um einen Scheingrund für die Unterschiebung zu gewinnen. Berta ist von der langen Reise erschöpft, sie bittet also die Freundin usw. Unser Romanschreiber wiederholt nun diesen Einfall, lärst aber die Ungereimtheiten weg. Wegen der Erschöpfung und noch aus einem andern Grunde, den uns der Dichter leider nicht verrät, beschliefst Berta auf den Rat ihrer Freundin, diese Nacht nicht beim König zu ruhen: ${ }^{2}$ gi deliberai che por cile soire la dame non albergast cun li roi por li civaucer ch'ele estoit stanche e por autre raison che li estoit". Wie oben gesagt, leitet die venetianische Handschrift allein die Mutter Rolands von der falschen Königin her und diese Genealogie wiederholt Marmora.

In der einen wie in der andern Version handelt die Verbrecherin auf eigene Faust. Marmora beseitigte sogar die Gestalt des Hofmeisters "bailo" als einen unbequemen Zeugen. Gaiete dingt selbst zwei Leute aus ihrem Lande, was beide Schriftsteller hervorheben, und trägt ihnen auf, Berta aus der Stadt zu führen und zu töten. In seiner Sorge um das Gelingen des Anschlags geht er soweit, dars er die Werkzeuge des Verbrechens beseitigt. Gaiete gesteht, dals sie die Schergen aus der Welt geschafft habe, damit sie das Geheimnis nicht verraten könnten. Davon ist zwar in der venetianischen Handschrift nichts erwähnt, der Dichter sagt gar nicht, was mit den Henkern Bertas geschehen, aber er bringt sie auch 
nicht mehr auf die Bühne, obwohl er bei der Konfrontation gute Gelegenheit dazu hätte. Aus diesem Schweigen zog der Romanschreiber offenbar den Schlurs, dals sie beseitigt worden waren und legte dieses Verbrechen der falschen Königin zur Last. Noch einige Motive aus dem franko-italienischen Gedichte fanden in dem Romane Marmoras Widerhall: das öffentliche Schuldbekenntnis, die freiwillige Demütigung der Verbrecherin Berta gegenüber, die Bitte um Verzeihung und Erlangung derselben. Die Szene ist in beiden Werken fast dieselbe.
Avant q'ela fust en le fogo bruxé,
Ella se fu molto ben confesé,
A tota jent dise li so peçé,
A Berta oit li perdon domandé,
Et ella li oit loialment doné.

(V. 1623-7.)

In dem Romane erzählt zuerst Gaiete Berta, wie das Verbrechen zustande kam. Das ist mit Rücksicht auf das spätere Zusammenleben der beiden Gatten notwendig; denn sonst könnte Berta meinen, der König habe bei dieser abscheulichen Tat mit Hand angelegt. Sodann bittet Gaiete um Verzeihung und erlangt sie mit Leichtigkeit.

Es scheint aus obigen Zusammenstellungen $2 u$ folgen, dafs Marmora seine Kenntnisse der Bertasage aus dem franko-italienischen Gedichte schöpfte, dals er es ganz bewulst umgestaltete, indem er die dort vorgefundene Fassung in eine andere verwandelte aus Gründen, die wir oben kennen gelernt haben.

Wollte jemand behaupten, dafs der Verfasser auch das Werk Adenets kannte, so kann man dagegen nichts einwenden und nichts hindert übrigens, dafs ein solcher Kompilator einer späteren Epoche auch eine zweite Quelle benutzte. Die Vergleichung der beiden Fassungen beweist, dafs eines der schönsten Motive, das unzweifelhaft Eigentum des brabantischen Dichters ist, sich in dem Romane vorfindet. Es ist bekannt, da/s nur bei Adenet Berta mit Entschiedenheit und Würde ihre Frauenehre vor der Zudringlichkeit des unbekannten Ritters verteidigt. Es ist, wie sich sofort zeigt, Pipin selbst, der später seiner Gemahlin wohl Dank weifs, dafs sie nicht dem Fremden zu Willen war. Allen anderen, also auch der ursprünglichen Version zufolge benimmt sich Berta, als ob sie nicht eine Königstochter, sondern die erstbeste wäre. Adenet, der kein Volkssänger war, sondern als Menestrel an dem Fürsten- und später an dem königlichen Hofe weilte, fühlts instinktmälsig diese Inkonvenienz und änderte entsprechend die Sage. Diese Szene erscheint nun bei Marmora, bei dem wir lesen: Li roi la prist por mans e la voloit fer seoir après lui; la dame soi tira arer e dit: "Non mi tochés che se sausés chi sui, vos non mi tocharizés por tot li trexor de Paris... Sire gi sui dame de li maor sire de cristientés e si sui file de li secund maor de cristientés." $\mathrm{E}$ alor 
li roi se marevoile fort e dist. „In cristientés non est maor signor de moi; dites moi, de chi estes vos mulier?" Respond la dame: "Gi sui mulier de li roi Pipin e file de li roi d'Ungarie". "Estes vos Berte?" „Oil, mon signor". Wir bemerken hier, dals die Szene zwar sehr lebhaft, aber etwas unnatürlich ist. Berta weifs, dafs es Pipin, ihr Gemahl, ist und benimmt sich so, als ob sie zu einem gewöhnlichen Ritter sprechen würde, wie das bei Adenet der Fall ist. Die Situation ist also ungeschickt erdacht und beweist damit die Nachahmung. Trotzdem kann man, ich gestehe es offen, in dieser Hinsicht nichts Bestimmtes sagen. Die Ähnlichkeit eines solchen Motivs sogar kann, wenn man keine anderen Beweise hat, zufällig sein. Man darf nicht vergessen, dals nach dem Verfasser des Romans auf Berta der Vorwurf lastet, dafs sie mit dem Freunde entflohen ist. Hätte sie sich nun Pipin ergeben, so könnte den König auch fernerhin der Verdacht beunruhigen, dafs sie eine leichtsinnige Person sei. Um eine solche, sie schädigende Meinung $z u$ beseitigen, konnte Marmora, auf ein analoges Motiv, wie wir es bei Adenet haben, verfallen sein, doch ganz unabhängig von ihm. Der Schlufs ist auch anders, als bei Adenet. Dort wartet Berta, bis sie Pipin vor dem Hofe und ihrer Mutter für die rechtmäfsige Gemahlin erklärt hatte, hier ergibt sie sich ihm gleich darauf und zwar auf demselben Wagen, der in dem franko-italienischen Gedichte erwähnt wird. Dort führte der Dichter als Scheingrund den Umstand an, dafs es sehr heifs war - es war nämlich Hochsommer - daher liefs Pipin den Wagen herrichten und schön mit einem Teppich schmücken, hier läfst er auch ohne Ursache den Wagen vorfahren, „denn heute", sagt er, "will ich kein anderes Gemach" (sic!).

Es ist also möglich, dafs die Ähnlichkeit zufällig ist, aber möglich auch, dafs Marmora das Werk Adenets kannte. Zur Unterstützung der letzteren Hypothese liefse sich auch noch anführen, dals bei ihm, wie bei Adenet, der Beschützer Bertas ein Mann aus niedrigem Stande, während er in dem franko-italienischen Gedichte ein Ritter ist. Es ist auch wahrscheinlich, dafs dem Verfasser von $M^{3}$ die Reali bekannt waren, da Marmora wie $R$ die Erzeugung Karl's am Flufse "Maine" (in $R$ "Magno") stattfinden lärst, in der ursprünglichen Version aber dies im Walde desselben Namens geschieht ("Maine" in T. "Mans" in $A$ ).

\section{Die Bertasage in Spanien.}

Die Version der Gran Conquista de Ultramar (L. II cap. 43) zeigt eine gewisse Verwandtschaft sowohl mit Adenet als auch mit dem frankoitalienischen Gedichte. Mit dem ersteren darin, dafs die Amme Berta einredet, es drohe ihr in der Hochzeitsnacht der Tod, und dals sie sich erbietet, an ihrer Stelle die eigene Tochter zu schicken. 
Das Motiv mit dem Messer, mit dem Berta die falsche Königin verwundet, erfährt in den drei Versionen eine allmähliche Verbesserung. In der Chronique saintongeaise bittet die Sklavin Berta, sie in den Schenkel zu stechen, was Berta - es ist nicht einzusehen, warum - tut. Bei Adenet verwundet sich die Sklavin selbst vor Bertas Augen und gibt ihr das blutige Messer zum Halten, das Berta ohne ersichtlichen Grund in die Hand nimmt; in dem spanischen Roman stürzt Berta, als sie sich betrogen sieht, selbst mit einer Schere auf die Verräterin.

Bei Adenet und in dem spanischen Roman ist keine Erwähnung von einem Wagen, auf dem Pipin mit Berta ruhte. In dem einen wie in dem andern ist die Rede von der Frau und zwei Töchtern des Beschützers Bertas im Walde, in beiden endlich erdenkt die Amme denselben Grund, als sie Blancheflor nicht in das Gemach ihrer Tochter einlassen will: die Ärzte hätten ihr das Licht verboten.

Andererseits verbleibt der spanische Roman insofern in näherer Verwandtschaft mit dem franko-italienischen Gedichte, als Pipin in beiden in Begleitung seiner Jäger in das Haus kommt, wo Berta verweilt, als dies vor der Entdeckung des Verrates geschieht, als schliefslich Blancheflor erst einige Jahre darauf kommt und die Sache aufklärt.

$\mathrm{Da} V$ und $C$ von $A$ unabhängig sind, so waren ihre gemeinsamen Züge, wenigstens manche, schon in der Urversion, die sowohl $V$ und $C$ als auch $A$ als Quelle diente. Jede der drei Fassungen hat unabhängig von der andern und gemäls der allgemeinen Tendenz, die Erzählung logischer zu gestalten, die gemeinsamen Momente entwickelt oder modifiziert und zufällig einige von denjenigen, welche nur einen doppelten Ausgang hatten, ähnlich gestaltet.

Das zweite spanische Werk, das die Sage von Pipin und Berta enthält, ist der Roman eines gewissen Antonio de Eslava. Das Werk unter dem Titel "die Winternächte" ist eine Sammlung von Sagen, unter denen sich auch die Geschichte von den Abenteuern der Prinzessin Verta oder Berta befindet. Die älteste bekannte Ausgabe ist aus dem Jahre 1609, herausgegeben in Pampeluna, die zweite aus dem Jahre 1610 in Barcelona, die dritte in Cordova. Das zehnte Kapitel 1 mit der Überschrift ${ }^{\text {se cuenta el }}$ nacimiento de Carlo Magno Rey de Francia y Emperador Romano" gibt eine Bearbeitung der ersten 17 Kapitel des VI. Buches der Reali de Francia.

1 Eine Inhaltsangabe veröffentlichte Gabriel Henri Gaillard in seiner Histoire de Charlemagne, Paris 1782, B. III. S. 369 ff., eine genauere Analyse Ferd. Wolf, op. cit., S. $45 \mathrm{ff}$., und eine Paraphrase Barthélemy in der Revue Britannique, 1868, S. $489 \mathrm{ff}$. 


\section{Die Bertasage in Frankreich.}

Übergeht man die wenigen Anspielungen auf unsere Sage, die schon in dem letzten Viertel des XII. Jahrh. auftreten, so ist die erste eingehende Bearbeitung derselben ein kurzer Abschnitt in der Chronik eines Mönches aus der Stadt Saintonge, die danach Chronique saintongeaise heilst und jetzt unter dem Titel Tote l'istoire de France ${ }^{1}$ veröffentlicht ist. Der Verfasser gibt den Inhalt einiger Volksdichtungen an; unter anderen erzählt er auch die Schicksale Berta's, ${ }^{2}$ aus denen wir die Gestalt der Sage in jener Zeit, d. i. ein halbes Jahrhundert vor Adenet, erkennen können. Die Gegenüberstellung der Version $T$ und des Adenet'schen Gedichtes zeigt, wie treu im allgemeinen der brabantische Dichter seiner Vorlage geblieben ist, obwohl er das ursprüngliche Werk erweitert und durch einige glückliche Züge bereichert hat. Die Chronique saintongeaise ist nach G. Paris in der ersten Hälfte des XIII. Jahrh. verfalst worden. ${ }^{3}$

Die zweite Chronik, die unsere Sage enthält, stammt von der Feder Philipp Mousquet's. ${ }^{4}$ Der Verfasser hat sein Werk noch vor dem Jahre $1244^{5}$ geschrieben, seine Version ist daher ebenso wertvoll, wie die vorhergehende.

Vor allem ist hier das Fehlen der Eigennamen auffallend. Man könnte meinen, Mousquet wisse nicht einmal, wie die Prinzessin, die Tochter Floire's, gebeifsen habe, da er sie kein einziges Mal nennt. Ebenso fehlt die Angabe, wo Floire König ist. Berta's grolser Fülse geschieht keine Erwähnung. Es handelt sich scheinbar um eine ganz andere Version, umsomehr, da die unklaren Verse:

\footnotetext{
Quar Pepins la sierve en ama,

Et la sierve prist la roïne,

Si la tramist en la gaudine.

Li roi par son consel l'osta,

En la foriest le coumanda

Mener et livrer à exil;
}

(V. $1985-90)$.

uns auf den Gedanken bringen könnten, der König handle ganz bewulst im Bunde mit der Sklavin. Doch das wäre ein irriges

1 New first edited ... by F. W. Bourdillon London. 1897.

2 Der Abschnitt, der unsere Sage enthält, findet sich in der Histoire littéraire de la France, B. XX, S. 702, mit einigen Ergänzungen bei G. Paris in der Hist. poetique de Charlemagne, S. $224 \mathrm{ff}$. nach beiden Hds. aber in der Tote l'istoire, S. 53-5. (S. dort die Einleitung von G. Paris p. X.)

S. auch Voretzsch, Einf. in das Studium der afr. Lit. S. 465 und Grundrifs der romanischen Phil. B. II, S. IoI3.

4 Chronique rimée p. p. Reiffenberg, Bruxelles. 1836 , B. I, S. $81-83$, V. $1968-2007$.

B Monumenta Germ., B. XXVI, S. $718 \mathrm{ff}$. 
Urteil. Die Unklarheit rührt bei Mousquet von seiner aufserordentlichen Bündigkeit her. Bedenken wir nur, dals der letzte Vers von der Verbannung livrer a exil (also nicht von der Übergabe Bertas an die Schergen, die sie töten sollen) spricht, und schon drei Verse weiter versichert uns der Chronist, dals sich die Schergen der Königstochter erbarmt und sie nicht getötet hätten. Allerdings nennt Mousquet hier weder den Namen Bertas, noch Ungarn, noch Blancheflor, aber ein paar hundert Verse weiter führt er, als er von dem Tode Pipins spricht, alle drei Namen zusammen an:

Et sa feme resqui lonc tans,
Bierte, ki fu fille al roi Floire
Et Blanceflores, ce dist l'estoire,
Et cele fu Bierte as grazs piés.

(V. 2335-39.)

So bestätigt die zweite Version den Abrifs der ersten, die Gemahlin Pipins, „Berte as grans pies", ist die Tochter Floire's und der Blancheflor. In dieser Form tritt uns die Sage von allem Anfang an entgegen, denn schon der um das Jahr 1170 schreibende Dichter behauptet, da/s „Berte as grans pies“ die Gemahlin Pipins und Mutter Karls des Grofsen war.'

Mousquets Chronik kann nicht von der Chronique saintongeaise stammen, da sie eine wichtige Einzelheit enthält, warum nämlich. Berta sich hat vertreten lassen, eine Einzelheit, die in $T$ fehlt und die wir bei Adenet vorfinden.

Der Verfasser des besten Bertagedichtes ist Adenet li Rois. Charakteristisch für sein Werk ist die behagliche, breite Schilderung und die Vorliebe für den Dialog. Die Aufgabe des Dichters fafst er sehr ernst auf. Er empfiehlt die Tugend, verdammt das Laster. Bei einer schlechten Person unterlärst er es nicht hinzuzufügen: Gott möge sie verdammen; bei einer guten: Gott möge ihr helfen. Als Tugenden gelten bei ihm Rechtlichkeit, Ergebenheit dem Herm gegenüber, Güte, Mut, Grolsmut, Freigebigkeit; als Laster: Verrat, Hinterlist, Grausamkeit, Habgier, Prellerei. Ein schlechtes Weib ist bei ihm zugleich auch wollüstig; Aliste versprach sofort ihre geheime Freundschaft dem Tibert; ein gutes Weib ist keusch und verteidigt mit ganzer Kraft ihre weibliche Ehre. Diese Tugenden sind nun bei Adenet im Besitze vornehmer Geschlechter, sie sind zum Teile eine angeborene Gabe, zum Teil das Ergebnis einer guten Erziehung, die sich in geschickter Ausdrucksweise und im Benehmen bekundet. Das Fehlen dieser Eigenschaften kennzeichnet Menschen niederer Klassen und heilst Gemeinheit. Courtoisie und vilenie sind die ständigen Gegensätze in dieser Zeit, die man schon von der Mitte des XII. Jahrh. an in den Werken der Hofepik sehen kann.

1 Du Méril, Floire et Blanceflor v. $8 \mathrm{ff}$. 
Natürlich bringt Adenet die Ansichten der höfischen Kreise zum Ausdrucke, in denen er erzogen wurde und lebte, die Ansichten seines Wohltäters und Erziehers Heinrich von Brabant, und auch die jener Damen, für die er den Cléomades schrieb. Wenn er es aber andererseits wagt, die allzu drückenden Steuern zu tadeln, die dem Volke während der Regierung Pipins durch die falsche Königin auferlegt sein sollten und noch auf seine Zeit andauern, so drückt er wohl die Klagen der niederen Stände aus und erhebt sich dadurch auf den Standpunkt eines höheren und doch unparteiischen Sittenrichters. Und ein solcher ist er in Wirklichkeit. Im Gegensatze z. B. zum Verfasser des Partínopeus de Blois, der den Damen ein schwelgerisches und ausgelassenes Leben anempfahl und es sogar sophistisch durch die Autorität der heiligen Schrift ${ }^{1}$ unterstützte, empfiehlt Adenet Demut, Sittenreinheit, Beobachtung der religiösen Pflichten, Gebet und Fasten. Seine Auffassung vom frommen Leben einer Frau, skizziert er in dem Portrait Bertas, während sie im Walde bei Simon weilt. Dieses strenge, hier entworfene Ideal, gereicht, man mufs es zugeben, dem Verfasser zum Lobe und hat dem Werke nicht geschadet. Berta ist uns sehr sympathisch, besonders in dem Augenblick, wo sie sich mit Würde gegen das ungestüme Begehren Pjpins wehrt.

Der Dichter hat ein lebhaftes Gefühl für die Schönheiten der Natur und für ihren Zusammenhang mit der Stimmung des Menschen. Daher lächelt, wenn es dem Menschen gut geht, ihm die ganze Natur zu; geht es ihm schlecht, wird alles düster und trübe. Adenet drückt das noch sehr primitiv aus, doch entdeckt er immer den Zusammenhang und versteht ihn geschickt darzustellen. Als man Berta in den Wald hinausführt, unterlälst der Dichter nicht zu sagen: „An diesem Tage war schlechtes Wetter, Kälte mit Wind und Regen", und diesen Satz, gleichsam einen klagenden Refrain, wiederholt er öfter, während sich Berta einsam in der Wildnis befindet. Als sie die Nacht überrascht, bemerkt der Dichter: ${ }_{n}$ Berta fürchtet die düstere Nacht". Als dagegen Pipin Berta im Walde begegnet und, von ihrer Anmut und Schönheit bezaubert, ihr seine Liebe gestehen will, sagt der Dichter: „Es war ein schöner, klarer Tag ohne Wind und Regen".

Adenet versteht es, zarte Gefühle mit aufserordentlicher Feinheit $\mathrm{zu}$ malen und seine Bilder vor Frivolität zu bewahren. Blancheflor nimmt Abschied von Berta, die sie in ferne Länder schickt. Wer weils, was für Schicksale ihrer harren? Das Herz der Mutter krampft sich zusammen bei dem Gedanken an eine vielleicht jahrelange Trennung. Anstatt ihren Schmerz in einem Strome rauschender Worte auszudrücken, wie das so mancher Dichter des Mittelalters tut, lärst Adenet die Königin einen Ring von Bertas Finger streifen: unter Tränen und Weinen wird sie ihn küssen.

1 Siehe die Studie ron M. Kawczyúski, Parténopeus de Blois, Krakau 1902. (Abdruck aus den Abhandlungen der philologischen Klasse B. XXXIV.) S. $72,94 \mathrm{ff}$. 
Cel anel de vo doit o moi enporterai,

En lermes et en plors souvent le baiserai

(V. 206-7.)

Oder ein anderes Bild. Eine Hofversammlung. An den Tischen sitzen die Herren. Unter ihnen ragt die Gestalt Karl Martells hervor. An seiner Seite die Königin. Da stürzt ein gereizter Löwe in den Saal. In grölster Bestürzung wenden sich alle zur Flucht. Der König zieht seine ohnmächtige Gemahlin mit sich fort. Aber Pipin zielt in aller Ruhe mit der Lanze nach der Brust des wilden Tieres. So führt uns Adenet seinen Helden vor. Dieser Augenblick würde ein ganz effektvolles Bild geben.1 Daher konnte auch der Dichter, nicht ohne einen gewissen Stolz, sagen, dafs seine Vorgänger die Geschichte der Löwentötung gar nicht gekannt hätten, dals er sie erst in dem Buche in St. Denis gefunden habe. Gekannt haben sie wohl auch andere, aber er war der erste, der es verstand, diese Szene lebendig darzustellen. Von nun an bleibt uns das Bild Pipins im Gedächtnis, seine kleine Gestalt tut seinem Ruhme keinen Abbruch, denn durch seinen Mut kommt er den Helden des Altertums gleich.

Wollen wir eine Idylle, wir finden sie am Ende des Werkes, wo Pipin Zelte und Nahrungsmittel herbeischaffen lälst, um einige Tage im Walde, in der freien Natur und auf der Stelle, wo ihm das Glück zuteil geworden, zuzubringen. Wünschen wir ein realistisches Bild? auch das findet sich. Erinnern wir uns der Königin Blancheflor, wie sie nach Paris reist, um ihre Tochter zu besuchen. Unterwegs hört sie lauter Flüche und Verwünschungen. Adenet beschränkte sich aber nicht auf Worte, er gestaltete seinen Gedanken plastischer in einem Genrebilde. Ein armer, verkommener Bauer wirft sich der Königin zu Fülsen, hält das Pferd zurück und erlaubt nicht weiter zu fahren. Auf Befehl der vermeintlichen Berta hatte man ihm das einzige Pferd, das er besals, genommen. Mit seiner Hilfe ernährte er sich und seine Familie, jetzt stirbt er vor Hunger. Führen wir diesen Abschnitt an, einen ähnlichen finden wir kaum in der ganzen damaligen Literatur.

Or s'en va Blancheflour, qui ot le cuer certain.

Enmi 82 voie encontre un paysant vilain;

Où qu'il voit Blancheflour, si la prent par le frain:

„Dame, merci pour Dieu, de vo fille me plain;

N'avoie c'un cheval dont gaignoie mon pain

Dont je me garissoie et ma femme Margain,

Et mes petis enfans, qui or morront de fain;

A Paris en portoie chaume et busche et estrain,

Soisante sous cousta, un an a en certain;

Or le $\mathrm{m}$ 'a fait tolir. Diex li doinst mal demain!

A meschief l'ai norri cest yver de mon grain,

1 In 2wei Hss. stellt die Anfangsminiatur eben dies Szene vor. S. $L_{i}$ Romans de Berte p. p. P. Paris S. LIIIf. und die Abbildung. 
Mais, par ce saint seignor qui d'Adan fist Evain, Je la maudirai tant et au soir et an main Que venjance en arai dou pere souverain."

Als die Königin mit dem armen Manne Mitleid fühlt und ihm hundert Sous geben lälst, verwandelt sich augenblicklich seine Stimmung, er küfst vor Freude den Steigbügel und die Riemen; "Gott mög' es dir vergelten, Herrin, dals ich jetzt gesund und fröhlich bin; bei den Reliquien des bl. Hermann, ich werde nie mehr Berta verfluchen" (V. 1770-74). Wie treffend ist hier der Charakter des Bauern erfalst!

Kräftig ist die Gestalt der hinterlistigen Amme entworfen. Mit starken Zügen prägt sie sich dem Gedächtnisse des Lesers ein und der Dichter hat der düsteren, ja, ich möchte sagen, dämonischen Farben nicht gespart. Als auf die Kunde von der Ankunft der Blancheflor alles den Kopf verliert, - Aliste rät zur Flucht, Tibert, die Schätze mitzunehmen, - da bleibt sie allein ruhig und will der Gefahr kühn in die Augen sehen. Sie werde nie zulassen, dafs Blancheflor ins Gemach trete, sie werde schon dagegen Mittel und Wege finden. Und sollte jene darauf bestehen, so wehe $\mathrm{ihr}$, mit einem Apfel oder einer Birne würde sie sie vergiften, sie habe es von einer Jüdin gelernt.

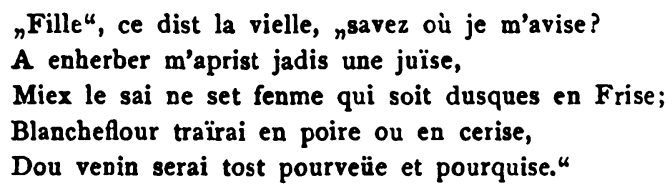

(V. $1829-33$.

Die Schicksale der ungarischen Königstochter überliefert uns auch eine Prosahandschrift, die gegenwärtig in der kgl. Bibliothek in Berlin aufbewahrt wird und aus der Mitte des XV. Jahrh. stammt. Feist's Annahme, dals dieser Prosaroman aus einer verlorenen Version $g$ geflossen sei, wurde von $\mathrm{Ph}$. A. Becker widerlegt. 1 Der Roman ist eine Paraphrase des Adenetschen Gedichtes. Was das Wappen betrifft, so scheint mir die Erklärung Feist's ganz verfehlt. Adenet fägt bei der Beschreibung dieses dem Simon vom Könige verliehenen Wappens hinzu, dafs es gegenwärtig noch im Besitze des Mannes sei, der es geerbt hatte.

Despuis l'a li lignages porté et portera.

Encor le porte cil qui l'eritage en a

$$
\text { (V. 3226-7.) }
$$

Nach Feist bezieht sich dieser Doppelvers auf eine in den Kreisen des Dichters bekannte Persönlichkeit. Wer mag das aber sein? Aufschlufs findet er in dem Prosaromane. Simon erhält dort den Beinamen le roy. Da nun Adenet ebenfalls den Bei-

1 Zeitschrift für rom. Phil.. B. XVI (1892), S. 2 I 5 ff. 
namen le roi hatte, hält er sich für einen Nachkommen Simons, und das Wappen, das er beschreibt, ist sein eigenes.

Eine überaus geniale Hypothese, die, wenn sie wahr wäre, gerade das Gegenteil beweisen würde von dem, worauf Feist hinsteuert. Überlegen wir nur: Wer hatte ein Interesse daran, Simon den Beinamen le roy zu verleihen? Wohl niemand anderes als Adenet, um sich und sein Geschlecht von dem Retter Bertas herleiten zu können. Wenn nun ein Prosaroman aus dem XV. Jahrb. diese Einzelheit bringt, so kann er sie einzig und allein dem Werke des brabantischen Menestrels mit dem Beinamen le roi entnommen haben. Dagegen kann man durchaus nicht begreifen, was den Vorgänger Adenets bewogen haben kann, Simon den für jeden Fall seltsamen Beinamen le roy zu geben.

Nehmen wir ferner an, dals jener unbekannte Autor, der Vorgänger des Adenet, ohne einen wirklichen Grund, nur so aus einer Grille, Simon den unverständlichen Beinamen le roi gab, mit welcher Hast würde in diesem Falle Adenet, wenn er das in seiner Vorlage gefunden hätte, es in seinem Werke vermerkt haben, eben zu dem Zwecke, um seine Herkunft zu verherrlichen. Sein völliges Schweigen lälst sich wohl nur schwer erklären. Dabei hat Feist bei der Behauptung, Adenet beschreibe sein eigenes Wappen, nachzuweisen vergessen, dafs die Menestrels überhaupt Wappen führten und dals das beschriebene Wappen gerade dem brabantischen gehörte. Bisher weifs man nur, dafs der Adel seit dem XII. Jahrh. Wappen gebraucht. Die Verse Adenets beziehen sich höchstwahrscheinlich auf das Wappen eines Barons aus der Umgebung des Fürsten, dessen Gunst Adenet zu gewinnen suchte.

Aus der ganzen Beweisführung Feist's könnte man höchstens den Schlurs ziehen, dals auf diesen Einfall schon vor fünf Jahrhunderten der Verfasser des Prosaromanes gekommen sei. $\mathrm{Da}$ er nicht wulste, auf wen sich der Doppelvers von dem Wappen beziehe, und ein Manuskript der "Berte aux grans pies“, wo Adenet mit einer Krone auf dem Haupte dargestellt ist 1 und den Beinamen le roi trägt, wahrscheinlich benutzte, auch Simon den Beinamen le roi anheftete und auf diese Weise mit dem Bande der Verwandtschaft den Verfasser der "Gutmütigen Berta“ mit dem Beschützer der Heldin verknüpfte.

Das bisher nicht herausgegebene Gedicht des Girard d'Amiens enthält die Abenteuer des jungen Karl, die offenbar mit der Geschichte seiner Mutter Berta und den Intriguen der falschen Königin in Verbindung stehen. Aus den zahlreichen, am Anfang verstreuten Anspielungen, die sich auf die Bertasage beziehen, kann man schliefsen, dafs Girard d'Amiens das Werk des Adenet als Quelle benutzt hatte. Übereinstimmend mit ihm nennt er Simon le voier:

1 Siehe die Abbildung bei Suchier Geschichte der französischen Literatur p. 205. 
Et comment puis apres sa fame retrouva

Chies symon le voier on il la recouvra.

Wie Adenet lälst er die falsche Königin, die auch Aliste heifst, in Montmartre einkerkern.

A mont martre ou lonc temps avoient demeuré.

Der Verfasser sagt selbst, dals er die Fortsetzung der Berta schreibe, ja, er übernimmt sogar völlig Adenet's Art der Versbildung; auf eine Strophe mit männlichem Reime folgt eine Strophe mit einem ebensolchen weiblichen Reime. Das allein sollte schon über die Abstammung der Version Girard's von Adenet entscheiden, umsomehr, da die Identität der Namen wie Elisette, Simon le voier die Annahme bestätigt. Feist wies das Werk Girard's d'Amiens der hypothetischen Gruppe $g$ zu. Wir können es ohne Zögern direkt von Adenet ableiten.

Die Chroniques de France sind ein umfangreiches Manuskript aus dem XVI. Jahrh. Das Werk dürfte nach G. Paris frühestens gegen Ende des XIV. Jahrh. entstanden sein. Die in dieser Handschrift enthaltene Fassung der Bertasage ${ }^{1}$ erinnert vollständig an die Version des Adenet. Feist reiht sie in die $g$-Gruppe ein, die hypothetische Version $g$ hat sich jedoch, wie wir oben gesehen, als Fiktion erwiesen.

Die Erzählung von den Schicksalen Berta's in $O$ ist sehr bündig gefalst; sie bringt keine Namen aufser Pipin und seiner Gemahlin, sowie zweier Söhne der falschen Königin, Hauffroy und Henry. Sie berichtet aber, dafs die Intrige gegen Berta "die Alte“ - „une faulce et mauldite femme vielle et envenimee en malice" geschmiedet habe. Sie schob ihre Tochter an Stelle Berta's unter, welche verbannt wurde. Dann aber nahm Pipin auf die Fürsprache der Barone und offenbar durch die Gnade Gottes Berta wieder zu sich und hatte mit ihr einen Sohn, Karl, einen mächtigen und tapferen Herrscher, der in der Jugend vor seinen Halbbrüdern aus der Heimat fliehen mufste.

Wie man sieht, erlaubt sich der Verfasser des Romans mehrere Veränderungen. Berta geht in die Verbannung, der König handelt im Bunde mit der Sklavin, die Barone legen für die verbannte Königin Fürbitte ein. $\mathrm{Da}$ sich aber diese Änderungen in keiner anderen Version finden, kann man annehmen, dals es willkürliche Neuerungen des Romanschriftstellers sind. Von den französischen Werken nähert sich die Version Valentin et Orson am meisten bald Adenet, bald den Chroniques de France. Abstammen kann sie so gut von der einen, wie von der zweiten Version. $\mathrm{Da}$ sich nun aber der Verfasser in der Einleitung auf die Chroniken beruft:

1 Der betreffende Abschnitt, wie auch der aus $O$ ist bei Feist abgedruckt 
,veritablement nous trouvons aux croniques anciennes que noble et vaillant roy Pepin si espousa et prist a femme Berte, dame de grant renommee qui en son temps eust et soufrit par envye grande habondance de pestillance et tribulation", so schreiben wir sie eher den Chroniques de France zu, umsomehr, da ihr Anfang auffallend ähnlich ist: „Le prince Pepin, pour la grant renommee ot a femme une moult noble dame, apelee Berthe ... la quelle ot moult d'aversite sans raison."

Und weiter lesen wir in der Chronik, dafs es der Alten im Einverständnis mit einigen ungarischen Grolsen gelungen sei, ihre Tochter, die Berta sehr ähnlich war, unterzuschieben; dals der letzteren niemand Glauben schenkte, als sie unter Schwüren beteuerte, sie sei Pipins Gemablin. Auf den Rat der Verräter wurde sie der Wache ausgeliefert, die sie in den Wald führen sollte.

Diese Version hat also schon im Keime die Entwicklung, welche ihr der Verfasser des Valentin et Orson gibt, dafs nämlich der König Berta nicht glaubt, sie sei die Tochter des Floire und seine rechtmäfsige Gemahlin. Daraus zieht der Romanschriftsteller seine Konsequenzen. Er lärst Berta in die Verbannung gehen und bewerkstelligt ihre Rückkehr auf die Fürsprache der Barone hin.

Das Miracle de Berthe ist der einzige dramatische Versuch im Mittelalter, der sich auf den karolingischen Sagenkreis bezieht. Die Frage, woher der Dichter den Stoff nahm, hat bereits Vogt 1 entschieden. Seine Untersuchung weist nach, dafs sich der Verfasser des Miracle ausschliefslich auf das Werk des Adenet stützt und ihm Schritt für Schritt folgt. Alle Personen, die im Adenetschen Gedichte auftreten, kehren mit denselben Namen in unserem Miracle wieder. Selten begegnet man einer so sklavischen Nachahmung, insofern es natürlich nicht eine Übersetzung ist. 2 Natürlich haben die Dialogform und der Umstand, dafs diese Stücke auf der Bühne gespielt wurden, viele Kürzungen nach sich gezogen. Manche Episoden hat der Verfasser gänzlich weggelassen, z. B. das Zusammentreffen Bertas mit den Missetätern, mit dem Einsiedler und der Bärin im Walde. Hie und $\mathrm{da}$ findet sich eine unbedeutende Änderung. Die Trauung findet nicht in Paris, sondern in St. Denjs statt. Nicht die Königin vertraut ihre Tochter der Margiste, die im Miracle wegen des volleren Reims auf Aliste Maliste heifst, an, sondern der König. Voigts Behauptung entgegen würde ich diese Personenvertauschung für keine besonders glückliche Neuerung erklären. Denn es ist viel natürlicher, dafs die Mutter ihre Tochter, die heiraten soll, dem Schutze einer erfahrenen Dienerin anvertraut, als

1 Die Mirakel der Pariser Hs. 819. Leipzig 1883. (Inauguraldiss. Grimma) S. 6 ff.

2 Trotzdem schwankt Feist: „Unumstöfslich sicher würde dies (Voigt's) Resultat erst sein, wenn nachgewiesen wäre, dafs $\boldsymbol{M}^{\mathbf{1}}$ mit $A$ in Gegensatz $2 \mathrm{u}$ $\&$ stände" (S. II); zuletzt erkennt er das Resultat Voigt's als wahrscheinlich an. 
der Vater. Das ist eine Sache, die Frauen angeht. Ganz merkwürdig klingen die Worte im Munde des Vaters an die Tochter:

Et je vous bailleray Maliste,

Ma serve, avec sa fille Aliste.

La mére vous enseignera;

La fille vous compaignera.

Die "Belehrungen“ aber, die Maliste Berta geben wird, werden für sie ganz verhängnisvoll. Der Verfasser drückt sich hier deutlicher aus, als Adenet, was eine grofse Heiterkeit bei den Zuhörern hatte hervorrufen müssen, besonders die naiven Bekenntnisse Bertas, wenn sie von der Alten hört:

\section{L'en m'a fait du roy mencion \\ Qu'il est d'une complexion \\ Qu'il n'est femme, s'a elle habite, \\ Qui n'ait du corps tant de labite \\ Qu'a peines se puist puis aidier.}

(V. $221-25$.

Berta entfernt sich erschreckt und beendet ihren Monolog:

... Mére Dieu, qu'est ce

Qu'homme peut telle chose avoir?

Pour riens ne le cuidasse, voir

Jour de ma vie.

(V. 270-72.)

\section{Die Bertasage in Niederland.}

Das niederländische Gedicht hat sich in mehreren Fragmenten erhalten, die zusammen kaum 150 vollständige und mehrere verstümmelte Verse enthalten. 1 Das ganze Werk dürfte 2000-3000 Verse gezählt haben, soviel man aus der Analyse der Fragmente und ihres Verhältnisses zur Vorlage schliefsen kann. Der Herausgeber schreibt die Autorschaft Diederic van Assenede zu, den wir schon bei den Untersuchungen über die Sage von Floire et Blancheflor kennen gelernt haben.

Es drängt sich die Frage auf, welches wohl die Quelle des niederländischen Dichters gewesen ist. Piot überlegt die Frage des Langen und Breiten und gelangt schliefslich zu keinem klaren Schlusse. Er vergleicht das niederländische Werk mit dem Adenetschen Gedichte und betont, dafs der Gegenstand derselbe ist. „Im ersten sowohl, wie im zweiten ist Berta die Tochter Floires und der Blancheflor und Pipins Gemahlin. Hier und dort gerät sie ins Unglück und irrt im Walde herum. Zuletzt finden sie die Verwandten wieder" (?). Doch sind auch bedeutende Abweichungen

1 Herausg. von $\mathrm{Ph}$. Piot. Bulletin de l'Académie de Belgique, Bruxelles 1875 S. II, B. XL p. $155 \mathrm{ff}$; auch v. Moltzer, Floris ende Blancefloer Bibl. van middelnederlandsche letterkunde, B. 23. Groningen 1879, S. $131 \mathrm{ff}$.

Zeitschr. (. rom. Phil. XXXV. 
vorhanden. Vor allem meidet der niederländische Dichter lange Beschreibungen und kürzt die Reden der im Gedichte auftretenden Personen. Bei ihm ist nicht jener deklamatorische Stil zu erkennen, der für Adenet so charakteristisch ist. Im ganzen, sagt der Gelehrte, verhält sich das niederländische Gedicht zu dem französischen, wie Reinaert de Vos zu dem französischen Renard. "Indem Assenede das französische Original umarbeitete, schuf er ein neues Gedicht, dem er durch Wendungen und darin enthaltene Ideen ein durch und durch flämisches Gepräge verlieh. Frankreich verschwand aus dem Gedichte vollständig, es ist kaum einmal erwähnt" (S. 161).

Das obige Urteil scheint mir ganz verfehlt. Assenede, von Natur ein rationalistischer Geist, war nicht imstande, wenn er überhaupt der Verfasser dieses Bertagedichtes ist, wie in der Umarbeitung "Floire et Blancheflor", so auch hier, zarte Gefühle, idyllische Naivität oder Anmut der Naturbilder wiederzugeben. Daher bei ihm die vielen Auslassungen und Kürzungen des Originals. Sonst folgt er treu der Vorlage, wie man es in den erhaltenen Fragmenten sehen kann.

Schon die Tatsache allein, dafs der Beschützer Bertas im Walde Simon heifst, läfst das niederländische Gedicht für eine Umarbeitung des Adenetschen halten. Aufserdem muls man den Umstand berücksichtigen, dafs dem niederländischen Dichter, der gegen Ende des XIII. Jahrh. schrieb, die Werke des brabantischen Menestrels wohl am besten bekannt und am leichtesten zugänglich waren. Adenet erfreut sich in jener Zeit einer grofsen Beliebtheit, Berta ist sein verbreitetstes Werk - sieben Handschriften sind auf uns gekommen. Es wäre daher nicht zu verwundern, dafs Assenede, der in jener Zeit den Roman Floire et Blancheflor umgearbeitet hatte, nun der Reihe nach die vaterländische Literatur durch eine Paraphrase der Adenetschen Berta bereicherte. Eine genauere Analyse der Fragmente bestätigt tatsächlich diese Vermutung. Der Dichter gibt zwar frei, aber doch ziemlich treu den Inhalt der einzelnen französischen Tiraden wieder, wie es Becker richtig gezeigt hat. 1 Nur an einer Stelle gestattet er sich eine Inhaltsänderung. Simon falst nämlich, als er Berta im Walde begegnet, Neigung zu ihr und macht ihr sogleich ein Geständnis. Natürlich will Berta, die doch später sogar die Bemühungen des stattlichen Ritters (Pipins) ablehnt, indem sie ihm erklärt, sie sei eine Königin, nichts davon wissen und fertigt den schon alternden Simon unverrichteter Dinge $a b . \mathrm{Ob}$ das ein glückliches Motiv ist, möchte ich bezweifeln, jedenfalls ist es eine Wiederholung der Szene mit Pipin, hier um so unpassender, als ein einfacher Förster den Don Juan spielt, eine Rolle, die gar nicht Menschen dieser Sphären entspricht.

Wir haben die Übersicht der langen Reihe von Werken, die sich auf die Bertasage beziehen, beendet und gesehen, dafs an

1 S. Zeitschrift f. rom. Philologie, B. XVI, S. 218 Note. 
der Entwicklung und breiteren Ausgestaltung derselben alle fünf Hauptliteraturen des Mittelalters beteiligt sind: sowohl die französische und deutsche, als auch die italienische und spanische. Jede von ihnen arbeitet die Sage einigemal um. Die Werke entstehen in einem langen Zeitraum von mehreren Jahrhunderten. Von der französischen Urversion, die ungefähr an den Anfang der zweiten Hälfte des XII. Jahrh. zu setzen ist, bis zu dem spanischen Roman Noches de invierno, der am Anfang des XVII. Jahrh. erschien, sind über 400 Jahre vergangen. Im nächsten Jahrhunderte führt Argenson die Sage durch die Romanbibliothek von neuem in Frankreich ein, doch nach der spanischen Bearbeitung, ferner Pleinchesne in Belgien in einer Schäferkomödie "Berthe"; andererseits gelangt die Sage durch die deutschen Chroniken in die Zahl der Jahrmarktsbücher, beginnt im Volke $z u$ kreisen und wird zu einem Märchen mit einer wunderbaren Grundlage.

Die Analyse der einzelnen Werke hat erwiesen, dafs von den 20 Versionen nur 6 von einander unabhänig sind. Alle anderen sind Werke $z$ weiter oder vielmehr dritter Ordnung; denn sie stammen bald von einer bald von zwei erhaltenen Gedichten, die, wie wir sogleich sehen werden, Umarbeitungen der verlorenen ursprünglichen Fassung der Sage sind. $\mathrm{Zu}$ den unabhängigen Versionen gehören: $T, S, M^{\prime}, A, V, C$.

Versuchen wir jetzt uns die Urversion zu vergegenwärtigen.

In der Einleitung vielleicht eine kurze Erwähnung, wie der kleine Pipin, gleich David, der den Goliath zu Falle gebracht, einen Löwen tötete (möglicherweise war diese Tat sogar ausführlicher erzählt, $M, A) .1$ Es folgt eine Hofversammlung $(V, A)$; die Barone raten dem König, ein Ehebündnis einzugehen $(T, A, V)$. Allgemein wird von der Schönheit der Berta mit den grofsen Fülsen $\left(F l . M^{1}, A\right)$, Floires Tochter gesprochen $\left(F l . T, A, C, M^{1}\right)$, des Königs von Ungarn $\left(F l . A, M^{1}\right) .^{2}$ Der Berta folgt als Dienerin $\left(M, T, M^{1}, A\right)$ ihre Amme $(T, C)$ und deren Tochter $\left(T, A, C, M^{1}\right)$, die der Königstochter sehr ähnlich ist $(A, C[V]$.). Die Amme erschreckt die unerfahrene Berta auf hinterlistige Weise $(T, C, A)$ durch die angebliche Gefabr, die ihr in der Hochzeitsnacht drohe $(M ! C, A)$, und unterschiebt an ihrer Statt die eigene Tochter $(T, A, C,[M])$. Frühmorgens begibt sich Berta in das Schlafgemach des Königs, um den ihr gebührenden Platz an seiner Seite einzunehmen; da verwundet sich die Tochter der Amme mit einem Messer in den

1 Mit $M$ ohne Index bezeichne ich die Fragmente Mainet's, mit $F l$. den Roman Floire et Blanchefior.

2 Nicht nur die "Chronique saintongeaise", sondern auch "Floire et Blancheflor" lärst Berta" vom ungarischen Könige abstammen. Dieses Werk ist bezeugt um das Jahr I 170 und der Prolog, der jene Erwähnung enthält, ist nicht eine Zutat, was aus der Tatsache hervorgeht, dafs er sich in beiden Handschriften vorfindet, die uvabhängig von einander von dem Urtext stammen, wie ich an einer anderen Stelle dargetan habe. S. Floire et Blancheflor, Etude de littérature comparée, Paris 1906, S. $5 \mathrm{ff}$. 
Schenkel, reicht Berta das Messer zum Halten und schreit, dals sie Berta töten wolle $(T, A, C)$. Der König erwacht und sieht Berta mit dem Messer in der Hand. (Unterdessen kommt die alte Amme herbeigelaufen und drängt Berta aus dem Gemache $(T, A)$. Die falsche Königin bittet Pipin, die Sache nicht ruchbar zu machen, denn sie selbst habe diese Sklavin mit sich gebracht und überdies sei die Amme unschuldig $A$ ). Auf Pipins Befehl liefert die Amme Berta den Schergen aus, die sie in den Wald führen und dort töten sollen $\left(T, M^{1}, A, C[V]\right)$. Dabei befiehlt sie ihnen zum Zeichen des vollstreckten Auftrags das Herz des Opfers zu bringen $(A, C)$. Von Mitleid ergriffen töten die Schergen Berta nicht, sondern lassen sie frei im Walde zurück $\left(T, M^{1}, A, C, V\right)$. Die Knechte bringen aber das Herz eines Tieres $(A, C$; in $A$ das eines Schweines, in $C$ eines Hundes). Berta irrt hungernd und frierend im Walde umher $(A$, $V$; in $C$ ist sie an einen Baum gebunden), endlich begegnet sie einem Manne niedrigen Standes $\left(T, M^{1}, A, C\right)$, [in $T$ einem Hirten (vachier), in $M^{1}$ einem Förster (forestier), in $A$ einem Forstverwalter (voyer), in $C$ einem Forstaufseher (montanero)], der bei der königlichen Domäne bedienstet ist $(T, A, C)$. Sein Name lautete vielleicht Simon (in $A$ Simon, in $V$ Sinibaldo), der seiner Frau Konstanze $(T, A)$.

Unterdessen gebiert die Tochter der Sklavin zwei Söhne $(M$, $T, M^{1}, A, C, V$ ), die die Namen Rainfroi und Heldri erhalten [in $M$ Hainfroi und Heudri, in $T$ Reimfré und Audri, in $M^{1}$ Raienfroit und Heldri, in $C$ Manfré und Carlon (!), in $V$ Lanfroi und Landris]. Es vergehen mehrere Jahre $(T, A, V)$, Blancheflor rüstet sich selbst, verwundert, dafs sie keine nähere Nachrichten von ihrer Tochter erhalte, zu einer Reise nach Paris $(T, A)$. Angst befällt die Frevlerinnen. Die falsche Königin stellt sich schwer krank. Sie heifst die Fenster verhängen und die Amme lärst niemanden, selbst Blancheflor nicht ein unter dem Vorwande, das Sprechen $(A, C)$ und besonders das Licht $(T, A)$ schade der Kranken; die Ärzte (fisiciens $A, C$ ) hätten es strengstens untersagt. Die Mutter verliert endlich die Geduld und dringt mit Gewalt in das Gemach $(T, A$, $C, V)$; Unheil ahnend reifst sie die Fenstervorhänge herunter, beschaut die angebliche Tochter und erkennt an den Fülsen, dals sie es nicht ist. Wie eine Löwin stürzt sie sich auf jene und bearbeitet sie mit den Fäusten $(T, A, C, V)$. Das Hofgesinde kommt herein, mit ihm kommt Pipin, alles kommt an den Tag $(T, A, C, V)$. Die Alte gesteht ihr Verbrechen ein und stirbt auf dem Scheiterhaufen $(T, A)$.

Blancheflor kehrt betrübt nach Ungarn zurück $(T, A)$. Einige Zeit darauf kommt Pipin auf einer Jagd $(T, A, M, C)$, bei der Verfolgung eines Hirsches $(A, C)$, zu der Behausung, wo Berta Schutz gefunden hat, und sieht dort die Königstochter $(T, C, V)$. Von heilser Leidenschaft entbrannt, begehrt er von Bertas Beschützer, dafs sie ihm willfährig sei, womit sich dieser einverstanden erklärt $(T, C, V)$ und für beide auf einem geschmückten Wagen ein Lager bereitet $(7, V)$. Das damals empfangene Kind erhält 
den Namen Charlemaine. Es kommt zur Erkennung. Erfreut schickt Pipin Eilboten nach Ungarn. Blancheflor kommt (zum zweitenmal $A$ ) nach Paris, wo die wanderbare Auffindung feierlich begangen wird $(A, C, V)$.

Das wäre in ganz allgemeinen Umrissen der Inhalt der Urversion und der Verlauf der Handlung. Die Fabel ist, wie man sieht, arm an Ereignissen. Daher war auch das Gedicht sicherlich nicht allzu umfangreich. Nimmt man als Grundlage die zeitgenössischen Gedichte aus der zweiten Hälfte des XII. Jahrh., das Durchschnittsverhältnis der beiden anderen Umarbeitungen des Adenet zu den erhaltenen Vorlagen und berücksichtigt dabei das franko-italienische Gedicht, so kommt man zu der Überzeugung, dals die ursprüngliche Fassung der Bertasage gegen 600-900 Verse zählen konnte. Man muls zweifeln, ob sie als solche ein Gedicht für sich bildete. Leicht konnte sie nur die Einleitung zu den Abenteuern des jungen Karl abgeben und den ersten Teil des Mainet bilden. In dieser ursprünglichen Fassung wiederholte sich eine Szene zweimal, nämlich der zweimalige Besuch der ungarischen Königin; aus diesem Grunde erwähnt $T$ nichts von der zweiten Ankunft, dagegen lärst $C$ die Erzählung kürzend, den ersten Besuch aus und verbindet die Entdeckung des Betrugs mit der sofortigen Rückkehr Bertas. Ähnlich handelte der franko-italienische Dichter, um eine offenbare Unwahrscheinlichkeit zu vermeiden. Bei ihm bleibt Berta nach der Begegnung mit Pipin im Schlosse des Sinibaldo noch vier Jahre; das hielt der Dichter für notwendig. Wäre Blancheflor früher gekommen und hätte den Betrug entdeckt, so müfste Pipin in jener Unbekannten sofort seine Gattin erkennen. Daher schob gleichsam der Verfasser der franko-italienischen Kompilation die Ankunft der ungarischen Königin vier Jahre auf. Nach der Entdeckung des Verbrechens erinnert sich Pipin sofort an die unbekannte Dame in Sinibaldo's Schlosse; zusammen mit Blancheflor begibt er sich zu Sinibaldo und bringt seine Gemahlin mit grolsem Pomp nach Paris.

Mousquet erwähnt in seiner bündigen Version gar nicht die Ankunft der Blancheflor; daraus folgt jedoch nicht, dals er ein Gedicht vor sich hatte, in welchem die ungarische Königin schweigend übergangen war. Denn wer sollte sonst den Betrug der Sklavin aufdecken? Nur die Mutter konnte, von Instinkt und Herz geleitet, darauf verfallen und in allen Versionen hat man diese Rolle für sie aufbewahrt. Bei Mousquet ist ferner die alte Dienerin, die Mutter der falschen Königin, mit keinem Worte erwähnt. Sollte auch sie in seiner Vorlage gefehlt haben und sollte darum jemand behaupten können, dals seine Quelle identisch war mit der des franko-italienischen Gedichtes? denn auch hier fehlt die alte Dienerin. Auf diesem Wege fortschreitend sollte man folgerichtig der Version, die der Stricker kannte, nicht nur Blancheflor und die Amme, sondern auch die junge Sklavin absprechen, da der Stricker in seiner noch knapperen Inhaltsangabe der Sage nicht 
einmal von der Tochter der Alten spricht. Wer hat aber dann Berta vertreten?

Es ist Zeit, auf diesem unzuverlässigen Wege der Vermutungen und Zweifel halt zu machen. Der Umstand, dafs zwei (sehr kurze) Versionen die Ankunft der Blancheflor nicht erwähnen, dagegen von den übrigen vier zwei vom zweiten Besuche, eine vom ersten und eine von beiden Besuchen berichten, zeugt, unserer Ansicht nach, sehr deutlich davon, dafs die Urversion beide Besuche enthielt. Der eine diente dazu, die Betrügerin zu entlarven, der zweite, die Tochter anzuerkennen $(A)$, (denn es konnten gewisse Zweifel obwalten, z. B. will bei Adenet Berta nicht gestehen), oder auch sich über die wunderbare Auffindung derjenigen $z u$ freuen, die sie schon seit Jahren als tot beweint hatte.

Wir haben diese Einzelheit ausführlicher besprochen, da es der einzige Anhaltspunkt ist über die Gruppierung der oben genannten sechs Versionen. Dem entsprechend, ob wir auf die Unterschiede, die wir in den besprochenen Werken in diesem Punkte antreffen, Gewicht legen oder nicht, wird die Antwort lauten auf die Frage, wie viele verlorene Urversionen es gegeben habe. Entscheiden wir uns für die erste Alternative, so werden wir zwei, mit der Zeit sogar mehrere solche verlorene Versionen erhalten. Es wird nur von der (Geschicklichkeit des Forschers abhängen, um zu der bestehenden Zahl der Versionen noch eine hypothetische hinzuzufügen. Nehmen wir die andere Alternative an, das heilst, dafs die einzelnen Werke bald infolge der Bündigkeit ihrer Fabel $\left(S, M^{1}, T\right)$, bald aus anderen Rücksichten (z. B. $\left.V\right)$ den Verlauf der Handlung in manchen Punkten modifizierten und einfach die erste oder zweite Ankunft wegliefsen, gelangen wir zu einem sebr einfachen Ergebnisse unserer Untersuchungen.

In der zweiten Hälfte des XII. Jahrh. entsteht ein Gedicht von den Schicksalen Pipins und Bertas, das eng mit Mainet, d. i. den Abenteuern des jungen Karl in der uns fragmentarisch erbaltenen französischen Version, der es als Einleitung diente, zusammenhängt. Durch mündliche Überlieferung gelangte dieses Gedicht nach Deutschland. Der Stricker hörte etwas von diesem Gedichte, skizzierte beide Erzählungen kurz aus dem Gedächtnisse und stellte diese Fassung an den Anfang des von ihm bearbeiteten Gedichtes des Mönches Konrad. Er nannte dabei keinen Eigennamen. Die angeführten wiederum entnahm er zum Teil der Kaiserchronik, wie Gertrud und Leo, die angeblichen Kinder des Pipin, teils entlehnte er sie von Personen des Rolandsliedes und übertrug sie auf $\mathrm{Ge}$ stalten der Bertasage wie Wineman, Rapot und Diepolt von Troys.

Der Chronist aus Saintonge und Mousquet, von denen der eine in Prosa, der andere in Versen die pseudo-historischen Züge von geschichtlichen Ereignissen entwirft, benutzen das Gedicht und geben dessen Inhalt knapper oder breiter in ihren Kompilationen wieder. Der spanische Verfasser der Gran Conquista de Ultramar gibt ebenso, wie Stricker, eine bündige Darstellung der 
beiden Sagen von Berta und Karl, hat jedoch das französische Werk vor sich. Daher bei ihm diese Genauigkeit in den Einzelheiten, die Abweichungen hingegen rühren aus der Tendenz des ganzen Werkes her oder sind manchmal das Ergebnis des Patriotismus des Verfassers, z. B. wenn er Floire nicht in Ungarn, sondern in dem spanischen Almeria herrschen läfst und dergl.

Dichterische Bearbeitungen der ursprünglichen Version gibt es nur zwei: das franko-italienische Gedicht und das von Adenet. Von den Abweichungen des ersteren haben wir ausführlich bei der Analyse dieses Werkes gesprochen, hier beschränken wir uns deshalb nur auf einige Worte. Die Stelle der alten Dienerin nahm die Mainz'sche Gräfin ein, die selbständig handelt. Die Erzählung von Berta bildet eine Episode der umfangreichen Kompilation und ist mitten in das Gedicht von Bovo eingeschoben. Diese zwei Umstände haben alle Änderungen verursacht und erklären dieselben. Alles Übrige ist eine Zutat des italienischen Dichters, die in keinem anderen Gedichte von Berta etwas Analoges hat.

Manche von den sechs Versionen wurden mit der Zeit Ausgangspunkte für andere Werke. In Deutschland übernimmt die kurze Erzählung des Strickers Heinrich von München, und ein Weihenstephaner Dichter, der Strickers Gedicht umarbeitet, dehnt sie zu einer grolsen Kompilation $(U)$ aus, indem er die Version durch Motive ergänzt, die aus anderen Sagen, z. B. der Pilatussage, geschöpft sind. Auf seine Version stützt sich die Weihenstephaner und die Wolter'sche Chronik wie auch der Verfasser der Zürcher Hs.; auf die Weihenstephaner Chronik wieder Ulrich Füetrer.

Das franko-italienische Gedicht bildet den Ausgangspunkt für zwei Versionen; die eine ist in Rafael Marmoras Roman Aquilon de Bavière, der gleichfalls im franko-italienischen Dialekt geschrieben ist; die zweite in dem pseudo-historischen, die Geschichte des Königshauses erzählenden Buche 1 Reali di Francia des Andreas Barberino," das auch von dem Adenet'schen Gedichte beeinflurst wurde.

Des gröfsten Erfolges erfreut sich natürlich das Werk des brabantischen Dichters, aus dem schöpfen: 1. Girard d'Amiens, 2. Chroniques de France, 3. der Romanschreiber aus dem XV. Jahrh. $(B)$, 4. der Verfasser des "Miracle de Berthe", 5. der niederländische Dichter.

Auf die Version der Chroniques de France scheint sich zu stützen der Roman Valentin et Orson.

Der Verfasser des spanischen Romans Noches de invierno wiederum bearbeitet die in den Reali di Francia enthaltene Fassung, indem er sie dem Geschmacke seiner Zeit anpalst.

Fassen wir das Resultat dieser Untersuchungen in ein schematisches Diagramm und vergleichen dasselbe mit den entsprechenden Figuren Wolfs und Feist's (S. 2 u. 3), so bemerken wir sogleich, worin das Resultat dieser Arbeit liegt, die auf genauer Analyse 
I 52 J. REINEOLD, UBER DIE VRRSCH. FASSUNGEN DER BERTASAGE.

der Versionen im Zusanımenhange mit den übrigen Teilen der Werkc und systematischer Erwägung des Wertes eines jeden M I.tivs für sich gegründet ist.

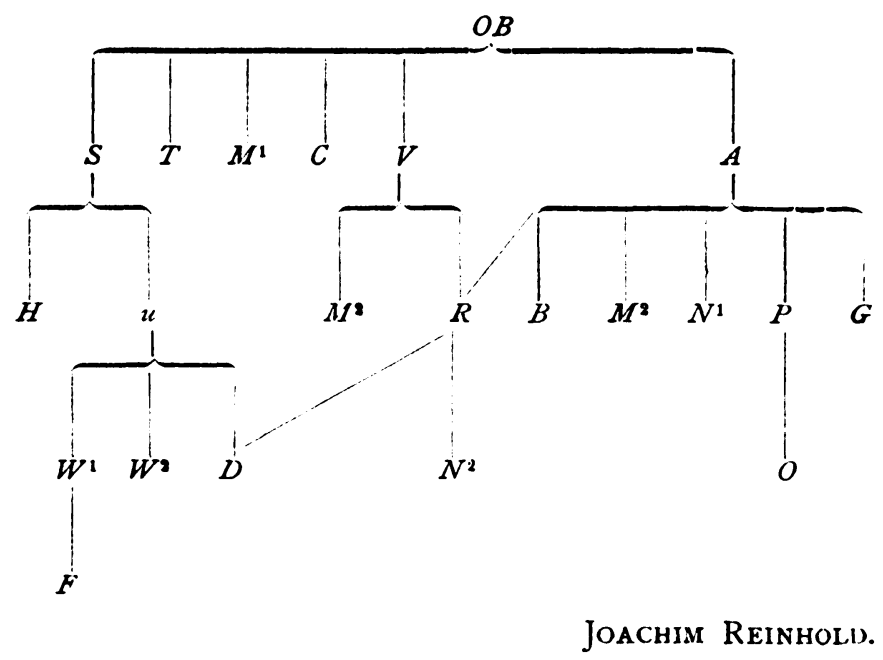

